\title{
Experimental Organism Syncytium
}

National Cancer Institute

\section{Source}

National Cancer Institute. Experimental Organism Syncytium. NCI Thesaurus. Code

C158331.

A single giant cell that is created from the fusion of mononuclear cells, or a network of cells that serve a particular purpose. 